\title{
Bioactive Glass Nanoparticles for Tissue Regeneration
}

\author{
Natalia Pajares-Chamorro a, Xanthippi Chatzistavrou a, ${ }^{*}$ \\ a Department of Chemical Engineering and Material Science, College of Engineering, Michigan \\ State University, East Lansing, MI 48824, USA \\ *Corresponding author. Email address: chatzist@msu.edu
}

\section{S1. Mesoporosity of M2-P1}

$\mathrm{N}_{2}$ adsorption and desorption isotherms were obtained at $77 \mathrm{~K}$ on ASAP 2020 Micromeritics machine. All samples were outgassed for $16 \mathrm{~h}$ at $200{ }^{\circ} \mathrm{C}$ under high vacuum in the degassing port of the adsorption analyzer. The specific surface area of the prepared samples was calculated from the $\mathrm{N} 2$ adsorption isotherms using the multipoint Brunauer- Emmett-Teller (BET) technique. Total pore volumes were estimated from the adsorbed amount of $\mathrm{N} 2$ at a relative pressure of 0.995 .

The isotherm of M2-P1 BGNs is type IV according to the IUPAC classification, thereby proving samples are mesoporous. The BET surface area and average pore diameter were calculated from $\mathrm{N}_{2}$ adsorption. BGN present a surface area of $21.95 \mathrm{~m}^{2} / \mathrm{g}$ and an average pore diameter of $18 \mathrm{~nm}$ with the presence of smaller size pores (i.e 1.7 and $3 \mathrm{~nm}$ ). The overall pore volume was $0.078 \mathrm{~cm}^{3} / \mathrm{g}$. The larger pores observed in BET (i.e $90 \mathrm{~nm}$ ) were correlated to the space between individual BGNs, demonstrating the presence of loosely aggregated nanoparticles.

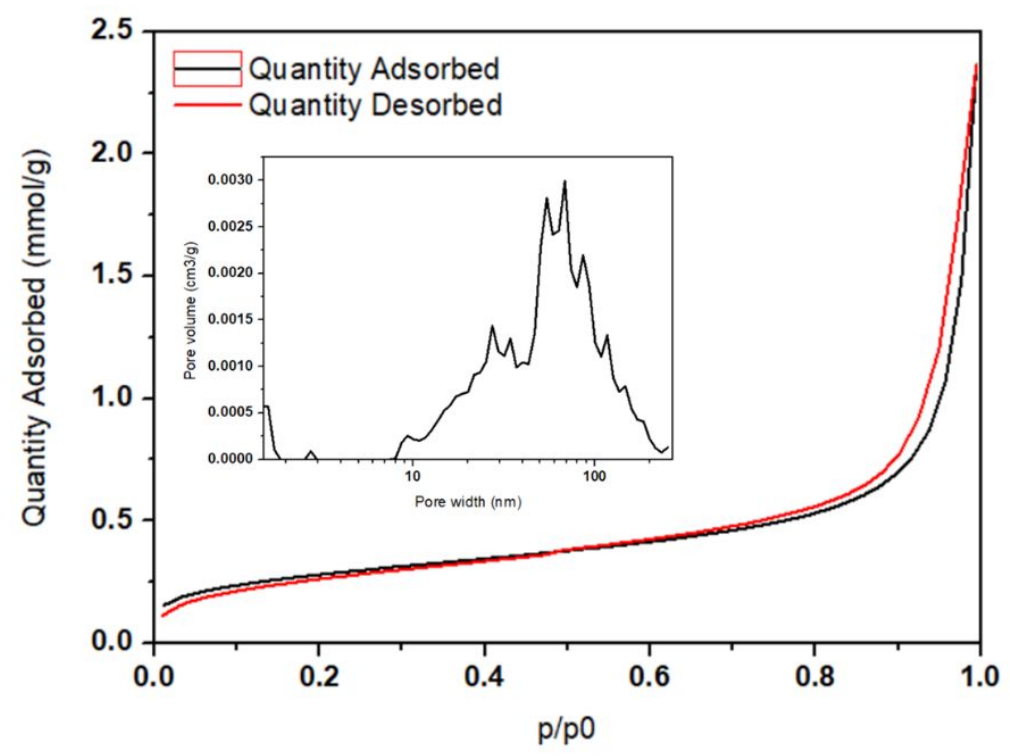

Figure S1: $\mathrm{N}_{2}$ adsorption-desorption isotherm of M2-P1 BGNs. Insert shows the pore with the distribution. 


\section{S2. Nanoparticle size and dispersity for M1-P2 and M2 protocols}

Particle size, size distribution (dispersity), and surface charge (zeta-potential) were also assessed with a laser dynamic light scattering (DLS) instrument (Zetasizer- nano series, Malvern Instruments Ltd). The BGNs were dispersed in Mili-Q water at a concentration of $1 \mathrm{mg} / \mathrm{mL}$ and sonicated for $10 \mathrm{~min}$ before measurements. Table 2 summarized the DLS data of M1 and M2 BGNs. Most values are within the range of the particle sizes measured in TEM. It is worth noticing that the distribution of size in M2-P1 and M2-P2 B\&C BGNS showed that $70 \%$ and $20-30 \%$, respectively, of the particles, appeared in aggregates and were not able to detach in the conditions of the experiment.

Table S1: M2 BGNs size and surface charge.

\begin{tabular}{|c|c|c|c|c|c|}
\hline & M1-P2 & M2-P1 & M2-P2 A & M2-P2 B & M2-P2 C \\
\hline Particle size & & & $120 \pm 10(30 \%)$ & $15 \pm 5(80 \%)$ & $22 \pm 6(70 \%)$ \\
and size & $500 \pm 30$ & $142 \pm 20(30 \%)$ & $200 \pm 30(60 \%)$ & $200 \pm 100(20 \%)$ & $300 \pm 50(30 \%)$ \\
distribution & & $990 \pm 100(70 \%)$ & $625 \pm 50(20 \%)$ & & \\
$(\mathbf{n m})$ & & & & & \\
\hline
\end{tabular}




\section{S3. Effect of stirring time in the incorporation of $\mathrm{P}_{2} \mathrm{O}_{5}$ in $\mathrm{M} 2-\mathrm{P1}$}

The proposed mechanism of incorporation of $\mathrm{P}$ ions was based on two parameters: hydrolysis rate and stirring time. To confirm this mechanism, the synthesis of M2-P1 BGNs was performed allowing a stirring time $\mathrm{X}_{1}$ of $4,8,12$, or $18 \mathrm{~h}$. Their SEM-EDS data was collected and compared to that obtained in BGNs stirred for $\mathrm{X}_{1}=24 \mathrm{~h}$. The concentration of $\mathrm{P}_{2} \mathrm{O}_{5}$ in mol.\% was calculated and the averaged of triplicate sample is summarized in Figure S3. Longer stirring times allowed TEP to hydrolysis further, releasing $\mathrm{P}$ molecules in the solution to homogenize with $\mathrm{SiO}_{2}$ tetrahedra. However, the nominal composition of M2-P1 BGNs was only achieved after $24 \mathrm{~h}$. The trend observed confirmed the slow hydrolysis of TEP in methanol, and thus, extended stirring was required to fully hydrolyze the reagent.

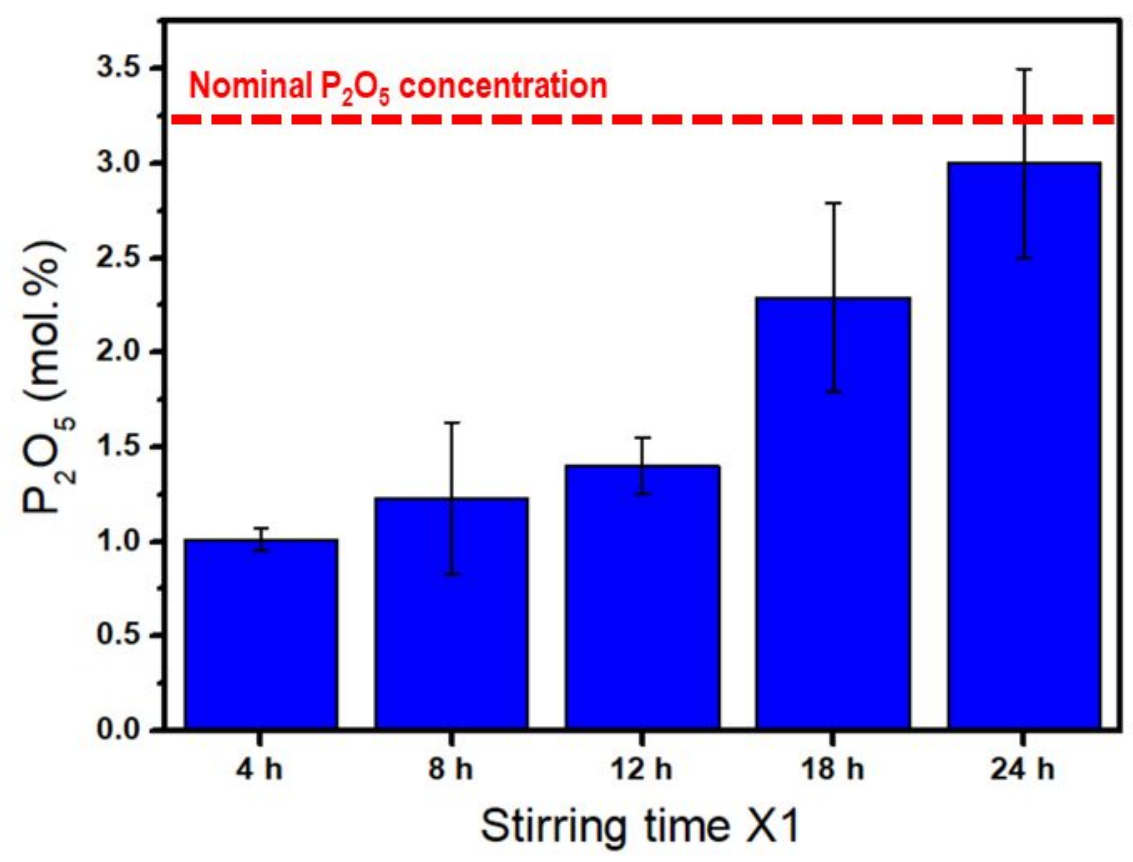

Figure S2: Effect of stirring time in the incorporation of $\mathrm{P}_{2} \mathrm{O}_{5}$ 


\section{S4. Presence and status of $\mathrm{Ca}^{2+}$ ions in M2-P1}

The proposed mechanism of incorporation of $\mathrm{Ca}^{2+}$ ions was further confirmed by SEMEDS and FTIR analysis on M2-P1 BGNs before calcination. The initial hypothesis described that the homogenization of the solution before catalysis allowed $\mathrm{Ca}^{2+}$ ions to get trapped within the silicate network. After calcination above 400C, these ions diffuse and modify the network. SEM-EDS was performed on M2-P1 BGNs after drying at 60C and proved that the concentration of $\mathrm{CaO}$ was around $35 \mathrm{~mol} . \%$, the same before and after calcination. FTIR showed the characteristic vibration of the silica network. The absence of the Si-O-NBO peak at $900 \mathrm{~cm}-1$, previously observed in Figure 2 for M2-BGNs, confirmed the silicate network was not modified before calcination despite the presence of Ca.

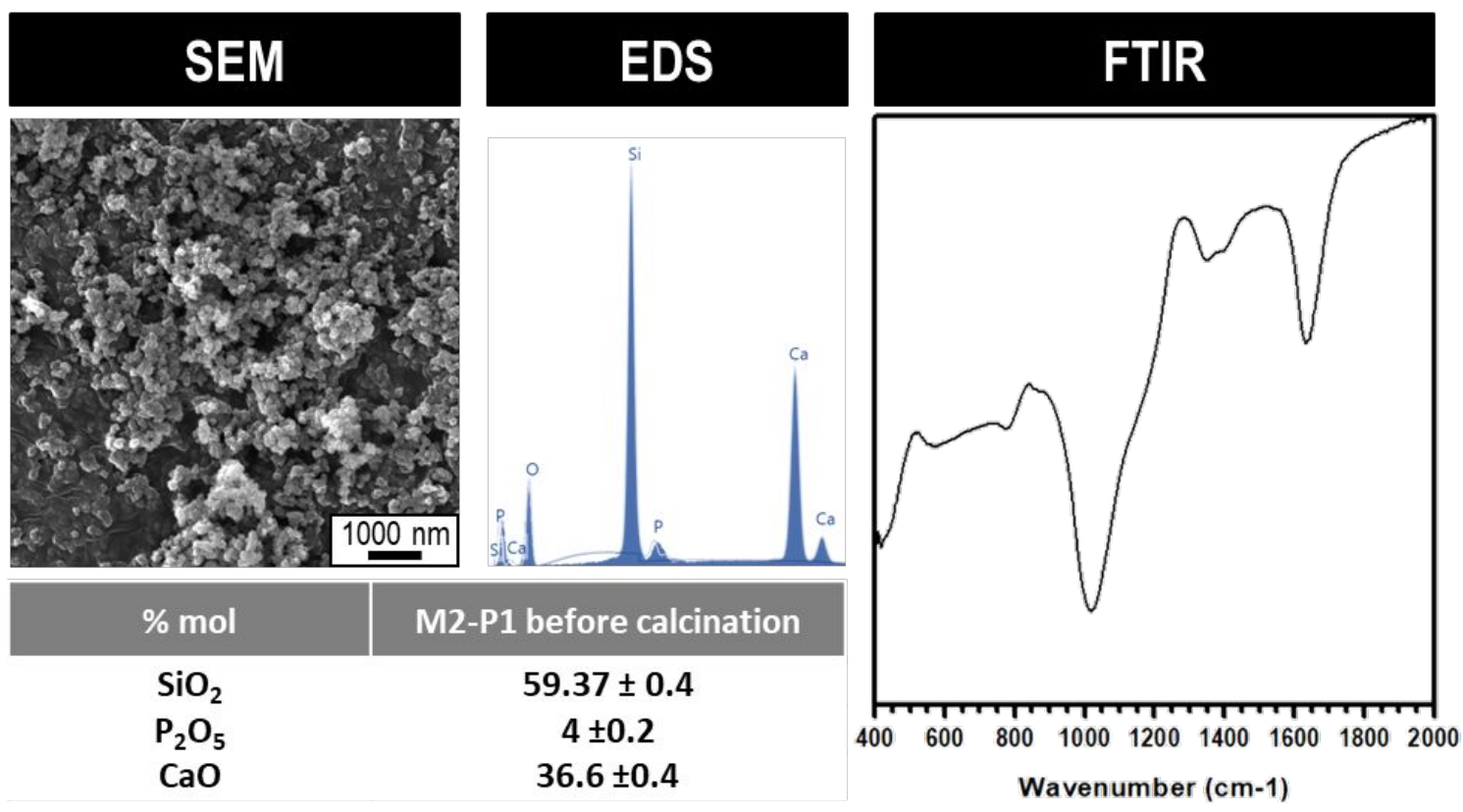

Figure S3: Elemental distribution and structure of M2-P1 BGNs before calcination. 


\section{S5. Effect of stirring time after particle nucleation}

One-step basic catalyzed synthesis protocols have often described the effect of different stirring times $\mathrm{X}_{3}$, collecting particles at different points after their nucleation. Specifically, extended stirring allowed the continuous and homogeneous growth of particles by the Ostwald ripening phenomenon. The protocol introduced in this work showed a neutralization of particle growth based on the stirring time after catalysis. In Figure S3, M2-P1 BGNs, collected after different stirring times, present similar particle size of about $90 \mathrm{~nm}$. Elemental analysis confirmed the overall composition was maintained during the stirring time. Thus, nominal composition was achieved prior to catalysis in the first nucleated BGNs thanks to the previous extended homogenization of the solution.
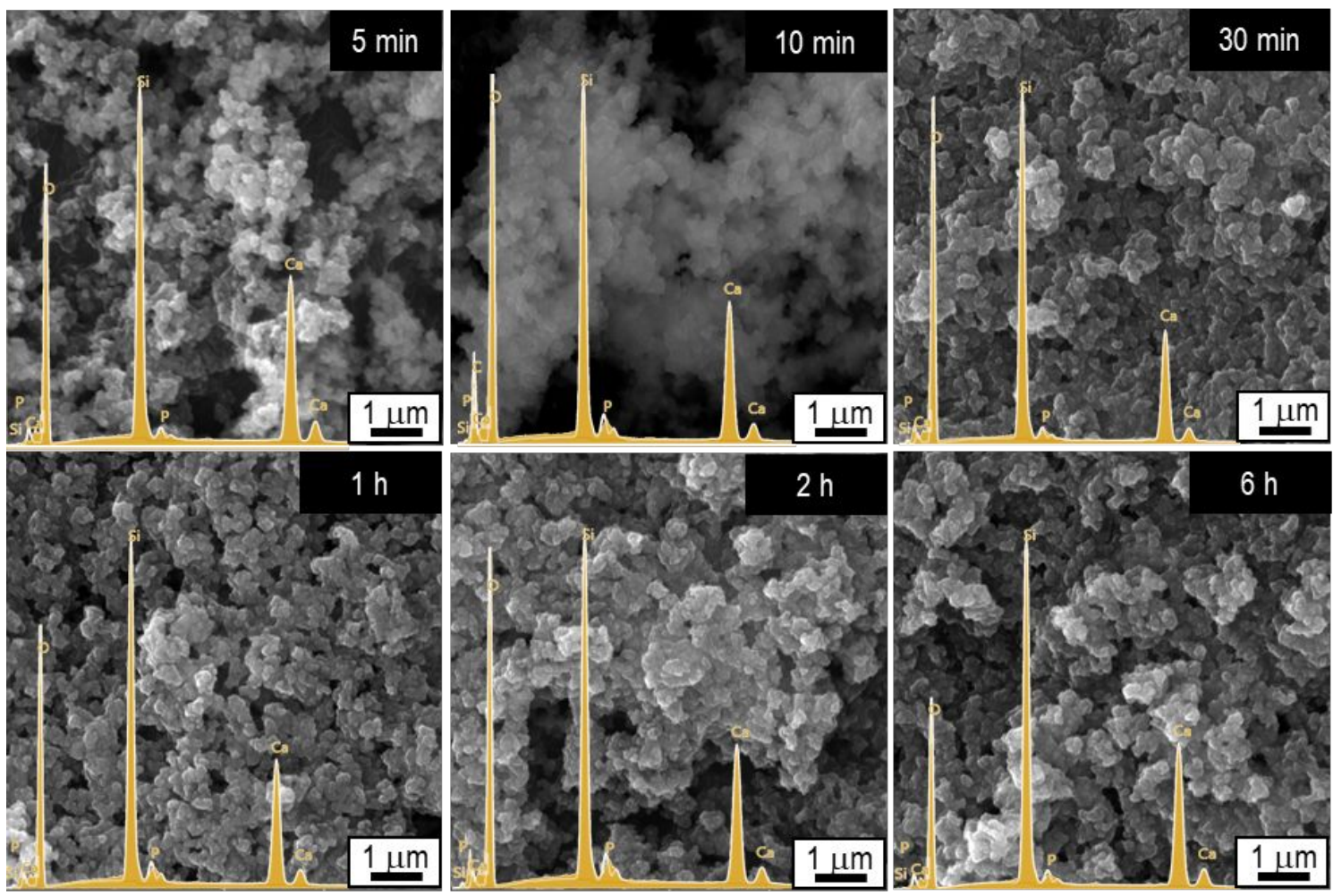

Figure S4: Particle size and elemental distribution of M2-P1 BGNs at different collection times after catalysis 